\title{
Alles, was Recht ist
}

\section{Nachbesserung erwünscht}

Wenn mal etwas schief geht mit dem Zahnersatz, dann hat der Zahnarzt das Recht, nachzubessern - ob dem Patienten das nun so richtig passt oder nicht. Das Bundessozialgericht (BSG) hat jüngst festgestellt, dass sich dieses Nachbesserungsrecht auch auf die Neuanfertigung des Zahnersatzes erstreckt - sofern dies dem Patienten zuzumuten ist. Das bedeutet: Falls das Vertrauensverhältnis zwischen behandelndem Zahnarzt und Patient nicht so zerrüttet ist, dass eine weitere Behandlung nicht mehr zumutbar ist, hat der Zahnarzt, der für den Fehler verantwortlich ist, das Recht, nachzubessern und Zahnersatz im Zweifelsfall auch neu anzufertigen. Im vorliegenden Fall hatte eine Patientin nach der Eingliederung einer Krone den Zahnarzt gewechselt. Dass die Krone mangelhaft gearbeitet war, stellte sich erst später heraus, die Patientin wollte aber nicht mehr zu ihrer früheren Zahnärztin zurück, die die Krone eingegliedert hatte. Die Patientin argumentierte mit mangelndem Vertrauen. Vor Gericht hatte dies allerdings keinen Bestand. Der Vertrauensverlust sei nicht nachvollziehbar, hielt nach mehreren Instanzen auch das BSG entgegen. Ein nachvollziehbarer Vertrauensverlust könne entstehen, wenn nach mehreren Nachbesserungsversuchen nicht das gewünschte Ergebnis erzielt werde. Dies sei hier allerdings nicht der Fall, da die Zahnärztin in der Gewährleistungsfrist nicht einmal die Gelegenheit zur Nachbesserung oder auch zur Neuherstellung bekommen habe. Az. B6 KA 15/16R

\section{Wunschmedizin abgelehnt}

Nicht alles, was medizinisch machbar und vielleicht auch wünschenswert wäre, wird von den Krankenkassen bezahlt. Und auch das Jobcenter springt bei arbeitslosen Patienten nur für die Übernahme der Kosten von "gesundheitsbedingten Mehrbedarfen“ein, wenn diese „unabweisbar" sind - also auf jeden Fall notwendig und unumgänglich - und die Kasse dennoch die Kosten nicht übernimmt. Dies stellte das Bayerische Landessozialgericht (LSG) kürzlich fest. Geklagt hatte eine Frau, die an einer seltenen Erkrankung am Zahnkiefer leidet, deren Behandelbarkeit nach aktuellem Stand der Wissenschaft nicht geklärt ist. Ob die von Spezialisten vorgenommenen Heilverfahren tatsächlich wirksam sind, ist bislang nicht nachgewiesen. Deshalb hatte die Krankenkasse der Patientin die Kosten der Behandlung nicht übernommen. Andere schmerztherapeutische Angebote lehnte die Patientin ab und forderte vom Jobcenter die Kostenübernahme der Behandlung durch den Kieferspezialisten. Das LSG Bayern lehnte wie schon das Sozialgericht Augsburg den Antrag auf Kostenübernahme durch das Jobcenter ab. Es fehle ein kausaler Zusammenhang zwischen Erkrankung und Notwendigkeit der Heilmittel. Ohne medizinischen Beleg sei die angewandte Therapie „Wunschmedizin, die vom Steuerzahler nicht finanziert werden " müsse, stellte das LSG fest. Grundsätzlich sei es allerdings nicht ausgeschlossen, dass das Jobcenter Gesundheitskosten übernehme - aber eben nur, wenn diese „unabweisbar“ seien.

Az. L7AS 167/17 BER

\section{Hier steht eine Anzeige.}

\section{을 Springer}

